\title{
The Synergy of Light and Life
}

Human Centric Lighting, a New Reality in Healthcare Environments

\author{
Rafael Lledó* \\ Polytechnic University of Industrial Engineers of Madrid, Spain \\ *Corresponding Author: Rafael Lledó, CEO Lledó Iluminación, of Madrid, Spain.
}

Received: October 03, 2019; Published: October 28, 2019

DOI: 10.31080/ASMS.2019.03.0451

\begin{abstract}
The environment in which we have evolved for millennia and the customs of our ancestors have marked our evolution and who we are. The buildings were created to protect us from the adverse conditions of nature. However, the reduction of costs, incorrect practices, and not remembering our body needs, have made us build buildings that harm us physically and psychologically. To have environments that facilitate our healing process, we must create hospitals that somehow bring us back to nature.
\end{abstract}

Keywords: Environment; Nature; Circadian System

\section{Introduction}

The earth rotates regularly every 24 hours producing day and night. Before there was life on earth, it already had this light/dark cycle. Our bodies cannot perform all of their functions at the same time. That is why nature provided us with the circadian [1] system that regulates our bodily functions throughout the $24 \mathrm{~h}$ cycle. A biological clock is only helpful if it can be synchronized to an environmental signal. The light/dark cycle is the most persistent cue in nature. Light is the primary marker of our Circadian System.

The circadian system interacts with most of the body's systems [2]

We have to mimic the light/dark cycle in hospitals to help patients with their conditions and the medical team to do their work

Provision of light [3] including its intensity, spatial distribution, color properties, the moment in time and its cycles of light and darkness all play a role in contributing to our circadian system synchronization and therefore our health and wellbeing.

We spend $90 \%$ of our time indoors, where we have low light during the day and too much at night. This causes our biological clock to be desynchronized, affecting us, particularly when we are healing at hospitals. This lifestyle is associated with epidemiological research on the population's health, such as sleep deficiency, SAD, ADHD, myopia, diabetes, bulimia, or bone strength.

The buildings we occupy, isolate us from nature affecting our biological clock synchronization and therefore our health and wellbeing. These affect our ability to recuperate from medical con- ditions, creativity, and social capacities, these last ones very much needed by the medical team.

If we do not take into account our biological needs during design, construction and use of buildings, this premises cannot qualify as healthy buildings

Momentum around Healthy Buildings is rapidly growing. Human Centric Lighting (HCL) together with other indoor environmental quality factors such as air quality and temperature, acoustics, biophilia or adequate space design, can help in creating Healthy and Sustainable Buildings.

Hospital buildings need to be renovated to adapt their installation to today's needs. Such as the new distribution of spaces, to accommodate modern medical equipment, disinfection or to create a more pleasant environment.

There is the opportunity to make new or renovated buildings taking into account our biological needs to fit for the $21^{\text {st }}$ century. And contribute to patients healing process and well-being and productivity of the medical team through HCL and other technical building systems.

This document is a compilation of facts, ideas, and suggestions gathered in scientific and technical literature, practical cases as well as own research.

\section{Circadian rhythm}

The Nobel Prize in Physiology or Medicine 2017 was awarded jointly to Jeffrey C. Hall [4], Michael Rosbash [5] and Michael W. Young [6,7] "for their discoveries (the mid-1980's) of molecular mechanisms controlling the circadian rhythm". 
In 2002 it was discovered that the morphology of the eye serves its functions.

Visible Light entering the retina excites the rods and cones. These photoreceptors send signals through the optic nerve to the visual cortex to create vision.

Blue light entering the retina excites the photopigment melanopsin. It is contained in the intrinsic photosensitive retinal ganglion cells (ipRGC). About $0.5 \%$ of the retinal ganglion cells function as a photoreceptor being the fifth photoreceptor (Cone S, M, L, rods and ipRGC). Some of the ipRGC send signals to inform the circadian system and the rest send signals to regulate the pupil dimension. The ipRGC responds to low frequencies of light with a peak at 480n.m [8]. Rather than encoding retinal images, they react to the diffused light arriving into the retina. Signals from the ipRGC reach the suprachiasmatic nucleus ( $\mathrm{SCN}$ ). It is the main and major biological clock in mammals and is responsible for the synchronization of the pineal gland to the light/darkness cycle through a polysynaptic pathway. The pineal gland uses and sends information to regulate the production of hormones as needed in the different stages of the day, modifying our physical and neurobehavioral capacities and the regulation of body temperature. It is also responsible for entraining our circadian system with the natural day/night cycle and pupil reflex.

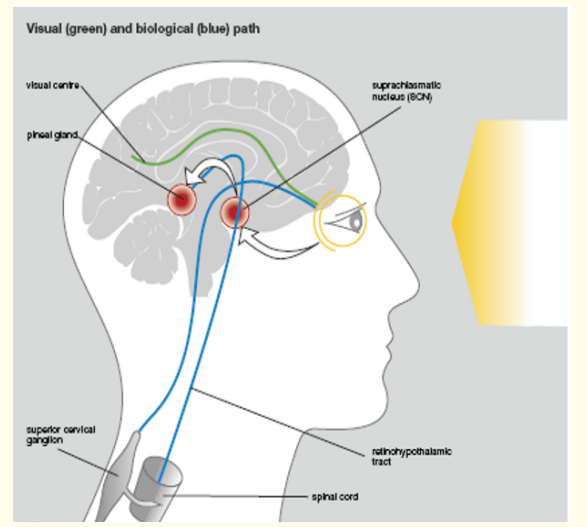

Figure 1

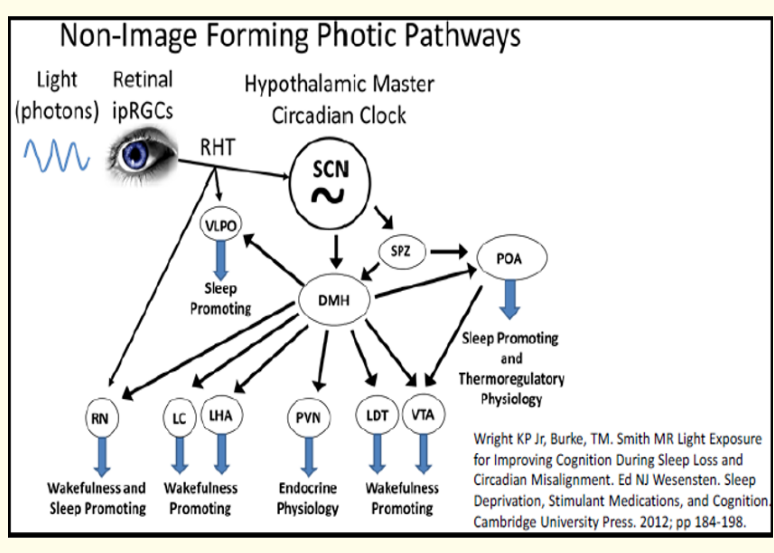

Figure 2
According to Lucas, et al. [9] in their publication "Measuring and using light in the melanopsin age", light is a potent stimulus for regulating circadian, hormonal, and behavioral systems. Also, light therapy is effective for certain affective disorders, sleep problems, and circadian rhythm disruption.

To measure if a light source (natural or electrical) produce a particular biological effect, it is necessary to determine its action spectra.

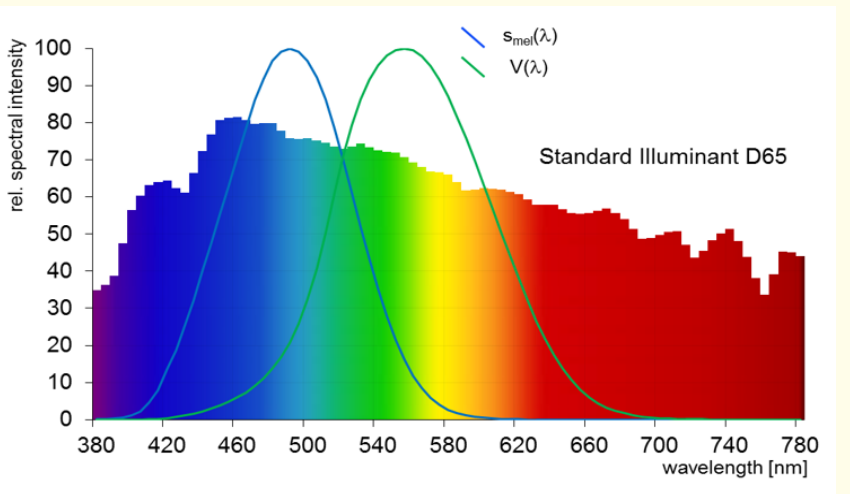

Figure 3

In figure 3, we can see the sensitivity curve of our visual system represented by the green line (maximum sensitivity at $555 \mathrm{~nm}$ ) and the melanopsin sensitivity curve represented by the blue line (maximum sensitivity at $480 \mathrm{~nm}$ ). The background colored image represents the chromatic characteristics of the Standard Illuminant D65 corresponding to the light produced by the sun in Brussels at noon in June. The standard and certification authorities use it as the natural light reference.

The biological clock gives the signal to start producing the daytime hormones and get us ready for our daily activities and to heal us from pathogens. Our body expects to receive bright white light (incorporating melanopsin light, blue line) in the morning. This signal synchronizes the biological clock by inducing small phase advances, needed because the intrinsic period of the clock is often slightly longer than $24 \mathrm{~h}$ and is therefore delayed. Late in the afternoon, warmer light and darker conditions during this time will help our clock to stay in synch. In the evening the biological clock gives a signal to produce the night-time hormones to help us relax and sleep and recuperate for the next day no light favors this process. Occupants/residents of a building do not receive the dynamic light that is produced in nature. Traditional indoor lighting cannot mirror the intensity, timing, color, and dynamics of natural light. HCL solutions can supplement natural daylight [10] resulting in beneficial [11] effects. However, a professional design is required as it is also conceivable that light can have adverse effects when misused. 
We can say "light is a drug", so we need to know how to administer it

Human-centric lighting in healthy buildings

Lighting Europe and IALD define HCL [12] as lighting, which supports health, well-being, and performance of humans by combining visual, biological, and emotional benefits of light.

HCL aims at providing the right light, at the right place and at the right time for the activities we carry out each day.

Electric lighting former solutions could not mirror the intensity, timing, color, and dynamics of natural light. HCL solutions can supplement natural daylight. A wide range of users can benefit from HCL, from patients, residents, and staff in hospitals and nursing homes, students and teachers in schools, employees in offices and manufacturing sites to residents in private homes.

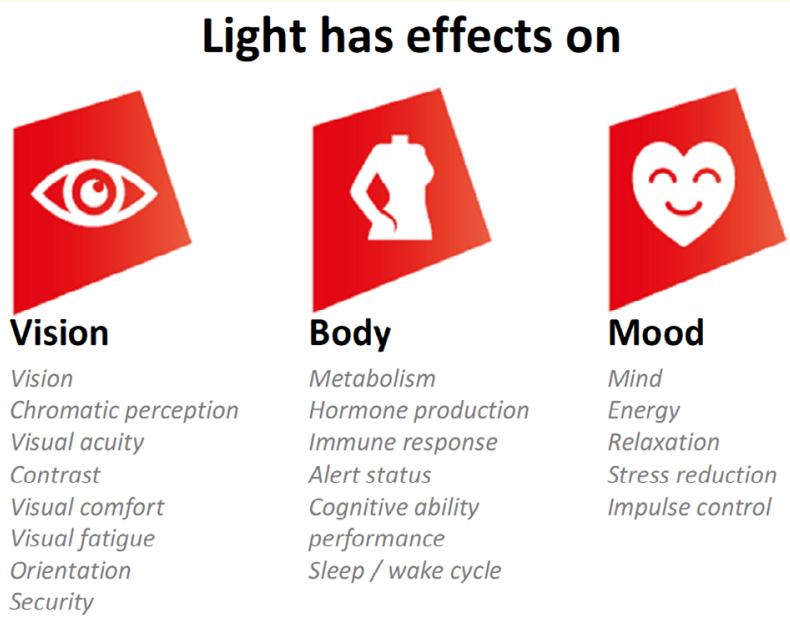

Figure 4

The introduction of the LED light source has revolutionized the lighting industry and opened many new opportunities not possible with previous technologies. Intelligent lighting systems can make indoor environments more attractive and functional, allowing light to be adapted to the needs of the users dynamically.

To make HCL part of a healthy building, the following elements should be considered:

- Lighting controls to adapt to the lighting level, timing, and color should be installed by default. Control technology should be user-friendly and easily (re)configurable.

- Lighting should become a layered solution, addressing the core issues of safety, task requirements, and occupant needs in a coherent and integrated manner.
- Energy efficiency should move from a predominantly "in the box" product focus to an "installed and operational" system focus, with energy savings coming from well-considered in-use operation within a building.

All HCL projects need to specify the right light for the activity, time of the day, age, gender, and race.

- In medicine, $1+1$ is not always 2

- In lighting and circadian lighting $1+1 \neq 2$

Mixing two white light sources don't produce the expected result in terms of color, color rendering, or melanopsin (circadian) stimulus.

Worm white + cool white $=$ pinkish - white and equal circadian stimulus as only the cool white portion.

People with different age and or gender needs a different amount of light for a specific activity and circadian impact.

There are new standards and regulations in the lighting industry, like DIN SPEC 67600 or CIE S 026. CIE [13] is the International Commission on Illumination. In this last one, you can read; "There is strong scientific evidence that light is not only essential for vision but also achieves important biological effects relevant for human health, performance and wellbeing" and establishes new metrics for the biological stimulation by light.

Using the right wavelength, we can be more efficient in the use of energy by not wasting on un-useful or damaging light. The concept of light efficiency can as we understand it today only refers to visual aspects of light and not to the circadian ones. With LEDs, we can be very precise in the production of a specific color. Together with the appropriate control systems, we can make the wavelength change over time adapting to our circadian needs, being very efficient for visual and non-visual aspects of light.

\section{Is blue light therapeutic or a hazard?}

In recent times it has been speculating that blue light hurts the eyes. It is not true, as long as the exposure time and the amount of radiation are controlled. However, if we are exposed to violet light of $400-450 \mathrm{~nm}$ with a peak at $430 \mathrm{~nm}$ for many years, it can damage our lens and consequently our visual system. On the other hand, circadian light with a peak at $480 \mathrm{~nm}$ is beneficial during the day because it feeds our circadian system without creating damage.

According to Professor Joan Roberts [14], we have to distinguish very well what kind of wavelength we use, both being very close in the spectrum. The right light is therapeutic, and the wrong one can cause severe problems in vision after very long-term of exposure. 


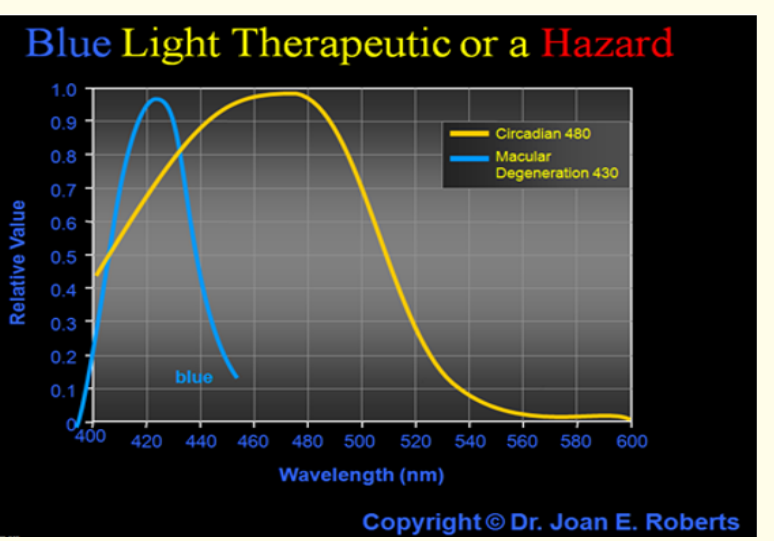

Figure 5

Clinical studies on circadian, neuroendocrine system and the therapeutic effects of light on humans, have predominantly used photopic light measurement (lux or candelas). These measurement systems are designed to evaluate the type of light that affects our vision system and not the circadian system, so they are inappropriate. Colorimeter or spectroradiometer are the right tools to measure the non-visual effects of light in humans.

Some sources radiate little or no light at the wavelength of $480 \mathrm{~nm}$ as can be seen in the attached graph. In the red (incandescent lamp), this light does not affect the circadian system but have other [15] biological effects. The green curve (fluorescent lamp), this light affects the circadian system but not too much. However, the blue curve, which corresponds to natural light, does have a blue component and influences the circadian system.

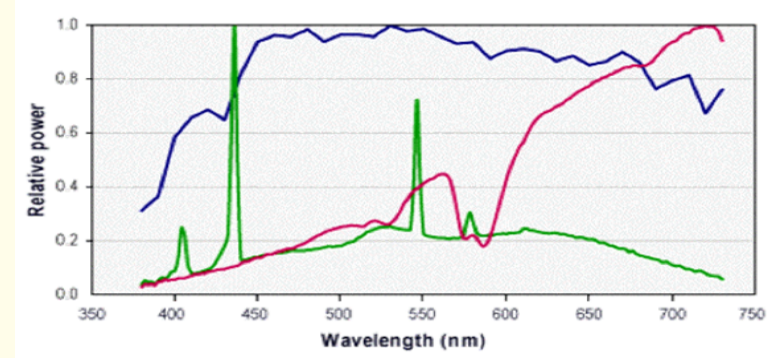

Figure 6

When clinical tests are done to measure the human circadian response to light, it is essential to know the detailed description about the spectral characteristics of the light arriving at the cornea of the patient, especially within the region between 460-500nm. The terms: bright light or low light, as we perceive it from our visual system are almost irrelevant biologically speaking as it has its own sensitivity courve.

Our body clocks control metabolism [16], contributing to the proper functioning of every organ in our bodies. The light/dark cycle is the main marker of our circadian rhythms. The circadian rhythm consists of physical, mental, and behavioral changes that follow a daily cycle. However, if we regularly bypass our natural day/night cycles by; working through the night, traveling over different time zones, or receiving too much white light before we go to bed, our body clock become disoriented and stop functioning correctly. It is what happens when we disrupt the circadian systems of patients in hospitals being in ICU or rooms using light or the lack of light in a given moment.

As we have seen, the variations in the dark/light cycles produce changes in our hormones and modify our cycle of sleep, blood pressure, metabolism, reproduction, and immune [17] response.

Affecting the level of activation of the body, memory, pleasure, alertness, muscle coordination, impulse control, desire for carbohydrates (appetite), converts body fat into muscle, increasing the sensitivity of senses, sexual activity and our response to stress. When we get older, our lens degenerates and becomes first yellow and later brownish — filtering the melanopic light — affecting us further getting fat without eating more or sleeping worse and less. While what we need is to sleep more.

As we eat food, our body receives tryptophan. Melanopsin [18] light triggers our circadian system to convert it to serotonin. In the absence of melanopsin light with the intervention of acetyltransferase and methyltransferase the serotonin becomes melatonin. When we receive light, the process stops producing new melatonin. The body discards the residues of melatonin through the urine.

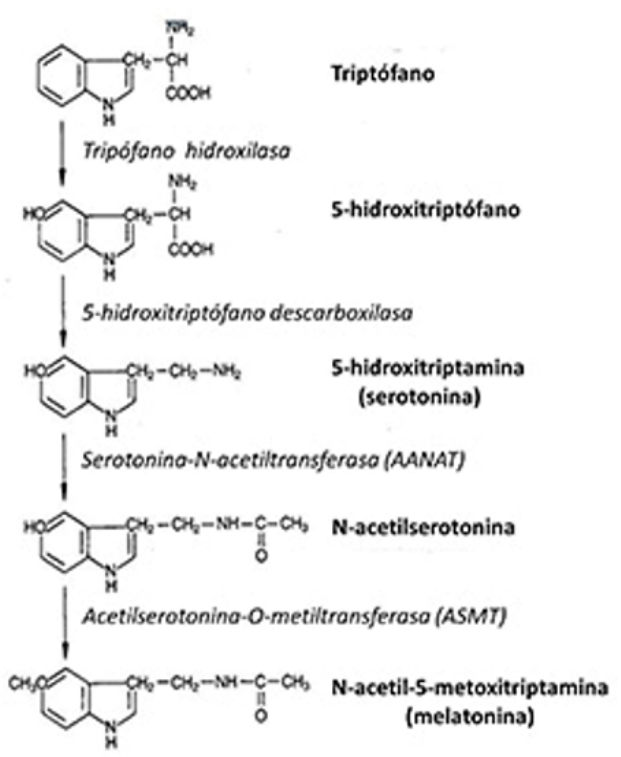

Figure 7

The more serotonin has been produced during the day due to the influence of sunlight or electric light that incorporates the blue component, the more we will have to produce melatonin during the night. 
The light mustn't damage or delay the sleep as it is when deep healing of the body is carried out.

If at the end of the day we receive blue light we will continue to produce dopamine instead of melatonin, which will delay sleep. This happens, for example, if we are looking at a computer screen, so it is advisable to use filters on the wavelength screens between 400 to $500 \mathrm{~nm}$ or at least reduce light intensity and use a black background. If we want to be active at night, we should never do it based on using blue light that makes melatonin to banish, so crucial for our health. We have to be activated by increasing the amount of warm light.

\section{Chronobiology}

Is the discipline of biology that studies periodic (cyclic) phenomena, or biological rhythms in living beings. Studies their temporal [19] organization, their alterations, and the mechanisms involved in their regulation. The central axis of chronobiology is based on the existence of endogenous biological clocks in organisms, from the molecular level to the anatomical level, which enable the execution of biological activity at a specific time point. In mammals, the biological clock is located in the suprachiasmatic nucleus of the hypothalamus. From there, the biological clock sends a "time" signal that reaches all the cells of the organism.

\section{The three basic cycles of Chronobiology}

Infradian rhythms (from Latin "infra", which means "below" and "dies", which means "days") are rhythms that last more than 24 hours. These rhythms are repeated at intervals of a few days, weeks, months, or even once a year. Therefore, a swing lasts more than 24 hours. Examples of this are seasonal rhythms, such as bird migration or lunar rhythms that follow the phases of the moon (about 29.5 days) and semilunar rhythms (about 14 days) that are normally associated with cycles of the tides. Even unpredictable rhythms, that is, those rhythms that have no correspondence in the environment, such as the reproductive cycle of women, are considered infradian rhythms.

Ultradian rhythms (from Latin "ultra", which means "beyond" and "dies", which means "day") are rhythms that last less than 24 hours. Many of the physiological functions of the human organism are an example of ultradian rhythm. These rhythms have multiple cycles in one day. An adult, for example, has an exercise and rest cycle of approximately 2 hours. Ultradian rhythms regulate physical, emotional, and spiritual functions. They often last for several hours and include food intake, blood circulation, hormonal secretion, different phases of sleep, and the body's performance curve. These processes lie in our body in millions of ways. Some last only a few seconds, such as breathing control, some up to thousandths of a second, such as brain orders or many of the processes that occur in the cells of the microcirculatory system.
Circadian rhythms (from Latin "circa", which means "around", and "dies", which means "day"): Rhythms that last approximately 24 hours. For example, the sleep-wake cycle of human beings. Many of the effects of circadian rhythms directly and immediately affect human beings; That is why they are the ones that are studied more thoroughly.

From the chronobiological point of view, the most important conclusion is that in our body, there is a true control panel that contains a series of genetically configured "biological clocks". First, our body follows an internal 24-hour cycle. The production of hormones depends fundamentally on the impulses sent by our biological clocks. Under the control of our brain, 150 messenger substances circulate in our blood that informs each organ about current conditions and prescribes specific reactions as needed. These hormones can have both a stimulating and calming effect. Our body temperature drops during sleep, blood pressure rises when we wake up, and so on. These rhythms are repeated every 24 hours, day after day, night after night. For this reason, scholars speak of a "circadian" rhythm.

At each moment, the circadian system transforms the internal actions of our organs into a unique and characteristic spectacle. From time to time, we can create unbalance of the circadian system, that creates a misalignment of your organs and body. It also seems that, with age, some of our biological clocks mark time more slowly, while others accelerate. Some tend to lag, and others even stop at some point. The organs develop independent rhythms and begin to manifest the first disorders. If left untreated, they can degenerate into real diseases. Science distinguishes around 80 conditions that can be attributed to sleep disorders.

The light-dark factor continuously adjusts our daily functions in a 24-hour rhythm. Within our body, there is a signaling hormone called melatonin. Around $11 \mathrm{pm}$ with the lack of melanopsin light (blue), there is a sudden increase between 8 and 10 times compared to normal values in melatonin levels. This is the signal for many organs that the time has come to slow down their activity and regenerate. However, this night surge of melatonin stops occurring in many older people. Many rhythms - including sleep, blood pressure, body temperature, and hormone secretion are left unchecked. In these cases, intelligent chronobiological help is needed. Some recent and exciting studies have resulted in valuable conclusions.

Researchers are beginning to understand at what time tumor cells tend to grow. Tumor cells are controlled by biological clocks different from those that regulate healthy cells. Therefore, it is of vital importance that the cytotoxins present in the drugs are introduced into the organ to be treated at the hours when their impact on the growth of the tumor is greater, that is when their effect on 
the rest of the cells is less harmful. Chrono Pharmacology or the search for therapeutic measures in harmony with our biological clocks is currently one of the fascinating medical research areas [20]. Gradually we realize many diseases are the result of disorders of our rhythms. This is the first step towards the principles of chronobiology.

Each cell produces highly specialized substances, such as the building blocks of amino acids. Once the ideal concentration is reached, production is interrupted. Within a few hours, the enzymes break down the substances. A new cycle begins. Some genes also called "clock genes", play the "on/off" switch function in these processes. They can be found in almost all tissues of the human body, and their impact is very multifaceted, sophisticated, and intelligent. Each part of the human body has receptors that capture information from messenger substances and neuronal stimuli. Its sensitivity is controlled by "clock genes". This also applies to the effects of vitamins, trace elements, and other biologically active substances, including medications. The SCN is the central point that controls the mechanism of actions that, as a whole, can be described as a control room where all the biological clocks are configured. From this control room, all the impulses that inform us of the passage from day to night, come from the outside world, and produce many rhythms adjustments in our body, synchronizing each other. Light is a powerful pulse generator. A person can recuperate faster from a heart attack thanks to the sun's rays that passes through the ICU window!

Chrono Pathology [21] addresses the issue of interrupted synchronization in vital biological processes. It describes different phenomena in the presence of anomalies in the chronological sequences: characteristics, causes, the importance of prognosis and diagnosis, and finally, the therapeutic implications. In each organism, the disease manifests itself at specific times. Annual and weekly rhythms have an impact on the susceptibility and frequency of conditions, the frequency of accidents, and the death rate. Deviations in biological rhythms are common. Blood pressure, for example, shows a good number of factors. The anomalies occur at certain times of the day, also in relation to the influence of the activity and rest phases.

Chrono Pathology studies these changing phenomena in relation to functional disorders of our organs, as well as diseases, with respect to the role of the day/night rhythm. The most common areas that highlight the need to deepen this science are "shift [22] workers" and "the jetlag", both characterized by the time lag. Chrono Pathology helps to identify the different phases of the deviation from normality. Recognizing time-dependent characteristics can be extremely important in the diagnosis and treatment of a disease. Smart anatomy is the basis of time-dependent drugs.
It is estimated that over one-third of the components of the genome are clock-controlled and over half of all drug-response pathways are clock-controlled

As we see, both daylight and electric light are NOT neutral to the human vision or circadian systems, as it affects our body in many ways.

Sleep-wake cycles are controlled by two processes: circadian and homeostatic

Circadian signaling is regulated by our biological clock every 24 hours, with the presence or absence of melatonin. It's telling our body ¡It is time to sleep or wake up! It is not what keeps us asleep.

Its production is regulated by the existence or not of natural light since this is the most reliable signal of nature. If we have not received enough light during the day, but at night, we stop producing melatonin at night delaying our sleep.

If circadian is out of sync, which commonly occurs with healthcare shift workers and extended-stay patients, sleep is disrupted.

The second process is affected by adenosine. It is a nucleoside regulating many physiological and pathological processes in the human organism. It is produced by almost all cells and is metabolized by adenosinedeaminase enzyme. Three types of adenosine receptors mediate the effect of adenosine. Adenosinergic system significantly influences the function of the cardiovascular system. Furthermore, it plays a key role in sleep homeostasis, in the regulation of bone metabolism and activation of the immune system. Adenosine mediates the effect of various hormones, but also adenosine itself has its own autocrine, paracrine, and systemic effects. Changes in endogenous adenosine levels, or changes of adenosine receptor sensitivity, may play a role in the etiopathogenesis of many diseases.

Adenosine is produced since we wake up "accumulating" during the entire time we are awake, increasing the pressure of sleep. It's what really makes us sleep.

Caffeine blocks and inactivate adenosine receptors, acting as a masking agent, decreasing sleep pressure.

When we receive the pressure of melatonin and adenosine, we fall to sleep.

Sleep disturbances in the ICU may lead to the development of delirium, prolonged stays, and increased mortality.

Lack of sleep can cause irritability and make focusing on even simple tasks difficult, affecting both caregivers and patients. 
According to Matthew Walker, the brain reactivity increases up to $60 \%$ with the lack of sleep [23] in a prolonged night.

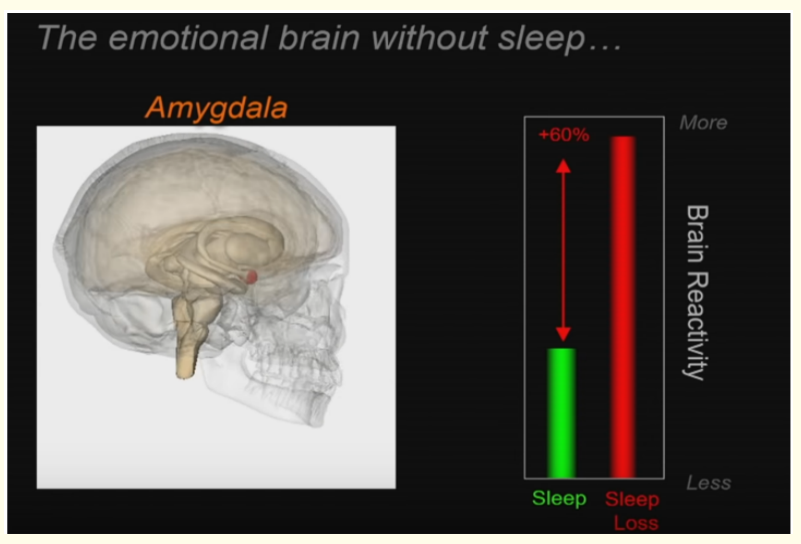

Figure 8

When you sleep 6 hours or less in a given night, you don't allow your body to heal itself. This can make you hungrier, weaker, dumber, sicker, less focused [24], and more prone to mood swings. In hospital environments, we are continually disrupting [25] the sleep of patients, through the noise, too much inadequate light, or medical supervision.

This must [26] change and consider and balance more carefully the needs of the patients.

A restful sleep to be fit and perform to the fullest

Sleeping well and enough is essential for our wellbeing, important for the regeneration of our body and daily performance. Unfortunately, we don't always enjoy a good restful sleep naturally. Many people around the world suffer from sleep disorders that not only substantially worsen the quality of their lives but can also cause health problems.

Sleep is an essential and vital need during which important processes occur. While we sleep, our body regenerates, our immune system stabilizes, cells are repaired and recover from the wear suffered during the day's activities. Also, while we sleep, everything we have learned is stored in long-term memory.

To improve people's well-being and effectiveness, we can act in two complementary ways at the circadian level:

1. Activating people during the day with enough radiation of light, around 480nm.

2. With its absence of radiation at this frequency at dusk, guaranteeing a deep and long enough sleep at night.

According to Professor Russell Foster, "Sleep is the most important specific experience of human behavior". although most of us do not give it the importance it deserves. Influential people have belittled the importance of sleep: Thomas Edison said; "sleep is a waste of time and takes us to cave days" or Margaret Thatcher; "sleep is of weeks".

iiiof course, they did not know the real value of sleep!!!

In the twentieth century, we use Edison's lamps to invade the night and occupy the darkness, and it seemed that sleep was our enemy. At best, we tolerate the need to sleep, and this is because of our profound ignorance about sleep.

We have the false belief that we do not do many things during sleep, and we are wrong! Sleep is a very important part of our existence. Neural scientists are beginning to explain why it is so important.

During sleep, some parts of our brain are more active than during wakefulness. A specific group of genes is only active when we sleep and are responsible for repairing and replacing cells. Sleep does not reach us from a specific part of the brain but from a series of interconnected parts that work as if they were a network.

By chosen to get insufficient sleep it seems we are accepting genetic manipulation to ourselves.

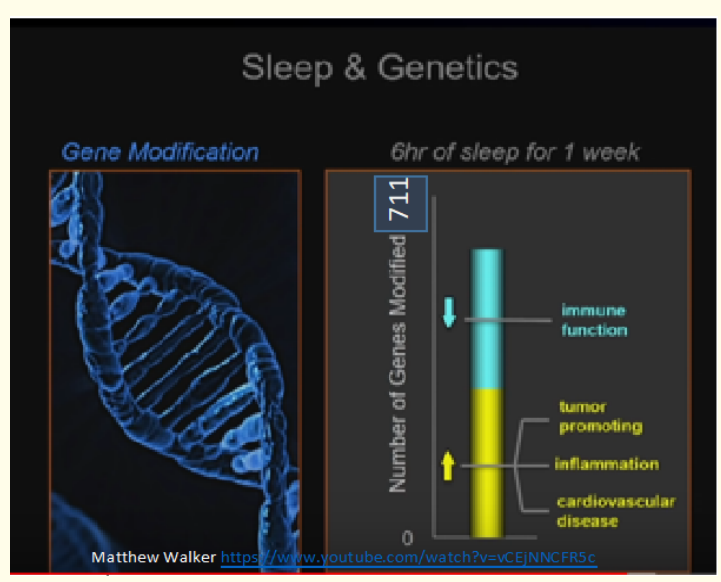

Figure 9

When sleeping only [27] 6 hours a day for one week, we experience modification in 711 genes. About half of them reducing our immune functions and the rest promoting: tumor, inflammation or cardiovascular diseases according to Matthew Walker.

Why do we sleep?

- To restore and rebuild our body and the brain itself, including metabolic functions.

- To reprocess the information received during the day by the brain and consolidate the memory, creating memories, 
learning, providing new solutions to solve complex problems, increasing these up to 3 times after a good night's sleep.

In the 1950 s we slept 1.5 hours more than nowadays.

If we sleep 5 hours, which is less than we should, we have $50 \%$ more chances of being obese, or increase stress, irritability, bad character, lack of memory and immunity and increase the need to smoke and drink.

If we don't get enough sleep at night, we still have the need to sleep during the day because we haven't completed the sleep cycle.

The complexity of all processes to produce the sleep/wake cycle makes it very vulnerable to its interruption.

As a doctor, you could prescribe to sleep as medicine since it is healthful free and enjoyable.

\section{When do we know we have slept enough?}

When we feel good and active when we do not need external stimuli when we focus and are attentive when we make the right decisions and are creative when we improve our training and are healthy.

The average person needs about 8 hours of sleep per day. But the amount required for each individual can only be determined by themselves, listening inwardly by analyzing and verifying the above.

What do we have to do to sleep better?

1. Receive enough circadian light during the day. Preferably in the morning.

2. Have physical activity and healthy life.

3. Have a healthy and balanced diet.

4. Have Nap of fewer than 30 minutes, finished before 4:00 p.m.

5. Do not take stimulants after the meal. Do not drink alcohol at night.

6. Do not eat at least two hours before bedtime.

7. Do not exercise or have activities that produce stress two hours before bedtime

8. Eliminate circadian light (include computers) 3 hours before bedtime.

9. Reduce the light 1 hour before (including the bathroom light to perform our grooming before bedtime).

10. Have regular schedules. Set the alarm to get up and another one iito let us know that it is time to go to sleep!!

11. The room temperature has to be cool.

12. Turn off the lights completely at night and do not send or check messages on mobile phones.
Solving insomnia problems are another matter.

In recent thorough studies, we have been able to verify that mental health and sleep are physically linked in our brain. Today, knowing it, we have new therapeutic tools to combat imbalances.

The fantasy writer Jim Butcher said: "Sleep is God, go worship" or Thomas Dekker 1572-1632 "Sleep is the golden chain that ties health and our bodies together".

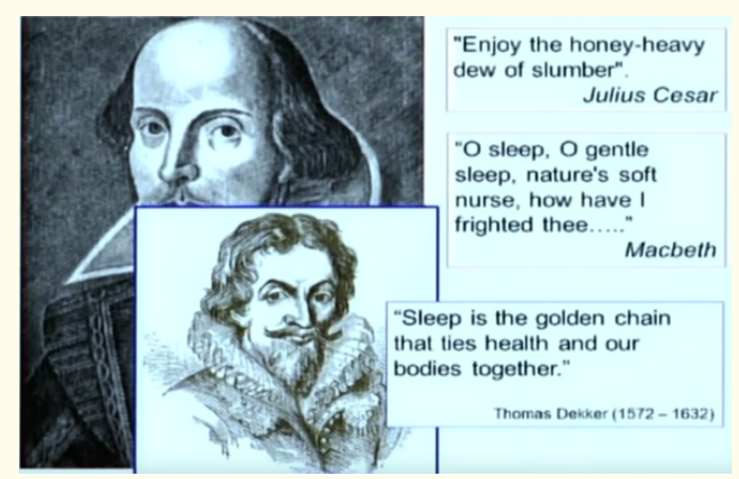

Figure 10

Sleeping not enough has negative effects on our immune system, favoring the development of diseases that at first sight can manifest in the form of a simple cold or with the symptoms of the flu. However, sleep disorders can eventually cause serious health damage. Chronic sleep disorders with an average of three out of seven nights increase the likelihood of gastrointestinal diseases, heart failure, stroke, diabetes, vascular diseases, and even cancer.

If sleeping were not so important to us, it would be the biggest mistake of our evolution since we should spend about 8 hours on it every day.

Sadly, human beings are the only species that will deliberately deprive themselves of sleep without legitimate gain (Matthew Walker).

A study by the EHJ (European Heart Journal) has shown that the risk of heart disease increases by $48 \%$ if you sleep less than six hours. Other studies have shown that depriving [28] the body of the necessary rest involves high blood pressure, clogged arteries, and heart failure.

A study carried out in Japan has shown, among other things, that there is a correlation between sleep disorders and diabetes, determining that high blood sugar levels for a long time (Hemoglobin Glucosilata, also HbA1c) show a clear connection with sleep disorders, such as waking up too early in the morning. It is suspected that poor quality sleep causes the release of the stress hormone (cortisol), which favors the development of diabetes. Last but 
not least, not getting enough sleep can cause cancer. The cases in which sleep disorders can be attributed to work in shifts are the most serious: in 2007 the International Center for Research on Cancer of the World Health Organization classified work in night shifts among the factors "probably carcinogenic" In particular, an increased risk of developing cancer has been observed among health personnel and flight attendants, as a permanent lag in the sleep/wake cycle.

Besides melatonin, many plant substances have positive effects on sleep. That has been studied from a chronobiological point of view to be able to be optimally combined with any other natural and biological substance.

Substances with a positive influence on sleep to take at night

- Hops

- Valerian

- Melissa officinalis

- Passionflower

- $\quad$ Mint leaves

Substances with negative effects on sleep

- Caffeine

- Alcoholic drinks

- Nicotine

- Heavy dinners to digest

The nap is one of the best and most straightforward things we can do for our health, work performance, and general wellbeing. Scientific evidence has shown that naps can improve performance and wakefulness, even after a typical night's sleep. The body needs to recover after 8 hours of activity, so it produces a small amount of melatonin that makes us feel stunned.

The nap can be useful for our working life and improve the day, but if we don't do it well, it can make us feel bad, sleepy and show us hostile. The following guidelines can help avoid these adverse side effects.

In general, it is better to avoid waking up during the deep sleep phase. The ideal time for a short nap is less than 30 minutes or more than 60 minutes, which is when the body is not in a deep sleep upon waking. The unwanted area to wake up from the nap is 30 to 60 minutes.

It is also better to sleep when the circadian component accompanies, this happening about 8 hours after waking up in the morning. If you do it at the end of the afternoon, you will delay night sleep.

In addition, different types of naps are appropriate for different circumstances. Long naps of more than 90 minutes are suitable when you are deprived of night sleep or prophylactic when you want to prepare for sleep deprivation. Short 30-minute naps help improve performance and reactivate us. In a recent study, people who took naps of less than 20 minutes in the middle of the afternoon experienced an increase in work performance and rated themselves as less tired.

Albert Einstein reportedly slept for at least 10 hours per day nearly one and a half hours more than the average people in Europe or America today (6.8 hours). He also used to take very short naps and he said it help him to be focused and with more imagination.

Please silence and no light at night to have a good long repairing sleep [29]

Back to light

Removal of melanopsin light exposure at night allows for the production of melatonin and vasointestinal peptides and growth hormones (metabolism and repair).

But normally we do not do so, and we keep on using activating light at night, desynchronizing our body with the natural circadian dark/light cycle. As this cycle controls and modifies the sleep/wake process, blood pressure, metabolism, reproduction, and the immune response, we are creating additional problems to the patients at the hospital premises.

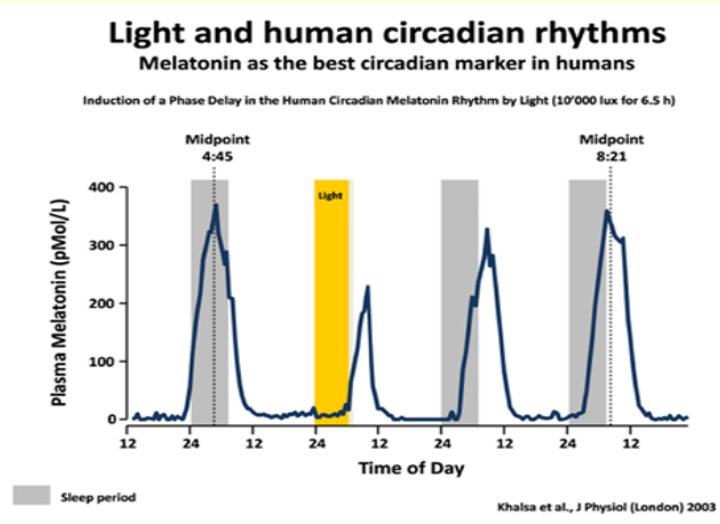

Figure 11

If we expose ourselves to light that incorporates blue component at the end of the day, we delay the onset of sleep, and this effect lasts several nights as we can see in the attached chart.

\section{Light is not neutral}

- It has either positive or negative effects on Human Health depending on the time of day, intensity and spectral composition.

- It is very important to take into account age, gender, race, and color preferences of the patient.

- Our needs of amount of light for circadian synchronization are higher than for visual stimuli

- $\quad$ Give me good light today because I want to sleep well tonight to be fit tomorrow!

According to a study published by the Brigham and Women's Hospital in Boston, sleep disorders and sleep deficiency are hidden costs that affect businesses across the United States. 70\% of Ameri- 
cans admit that they don't get enough sleep, and 30 percent of US workers. $44 \%$ of night workers report that they sleep less than six hours. In addition, it is estimated that between 50 and 70 million people have a sleep disorder, often undiagnosed. The costs attributable to sleep deficiency in the US were estimated to exceeded \$ 410 billion in 2015 , which is equivalent to 2.28 percent of gross domestic product.

The Brigham and women's hospital and Harvard medical school [30] in Boston, have developed the "Sleep Matters" initiative whose goal is to promote excellence in the treatment of sleep and circadian disorders to improve health, safety, and performance. Promote widespread changes in social norms that will engender a culture of sleep health.

"The promotion of healthy sleep benefits both people and businesses, improves the quality of life and longevity of workers while improving productivity and reducing health care costs", said Charles A. Czeisler, Ph.D., MD, FRCP, director of the initiative.

The occupational fatigue management program can increase awareness of sleep disorders, educate participants about the impact of reduced alertness due to sleep deficiency and teach anti-fatigue measures, as well as to detect sleep disorders to treat them".

Receiving enough quality light in the day helps us sleep at night as Steven W Lockley, PhD, a professor at Harvard Medical School explains: Light is not to generate our circadian rhythm, since it is endogenous in us, but to synchronize with the day, otherwise we would have a drift of about 30 minutes every day and in just 16 days we would not sleep until 8 in the morning of the next day, as is the case of people who are isolated from the daylight cycle and of society.

Not being correctly synchronized causes us problems in sleep, fatigue, reduction in our performance, mismatches in the production of hormones producing metabolic imbalances.

When exposed to circadian light in the morning we increase [31] cortisol, serotonin, Gaba and dopamine levels, modify the synthesis of the hormone that stimulates the follicle (FSH), the gastrin peptide (GRP) is released neuropeptides and TSH (metabolism).

If our body is in sync with the light/dark cycle, it will react, as indicated in the figure 12 .

The medical crises are also circadian as we can see in the figure 13.

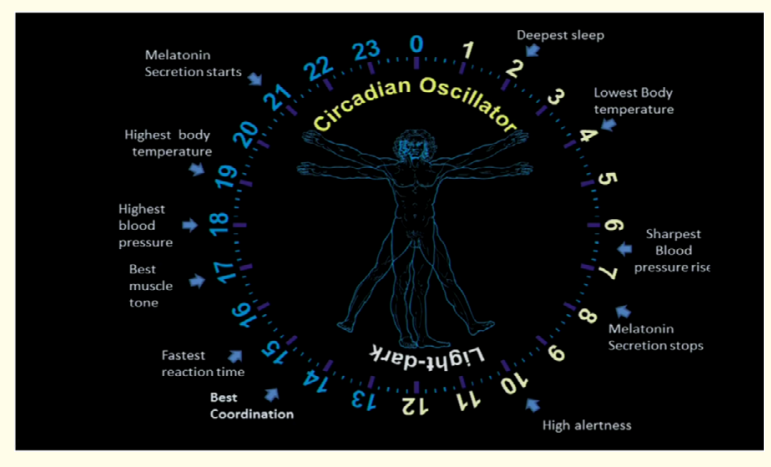

Figure 12

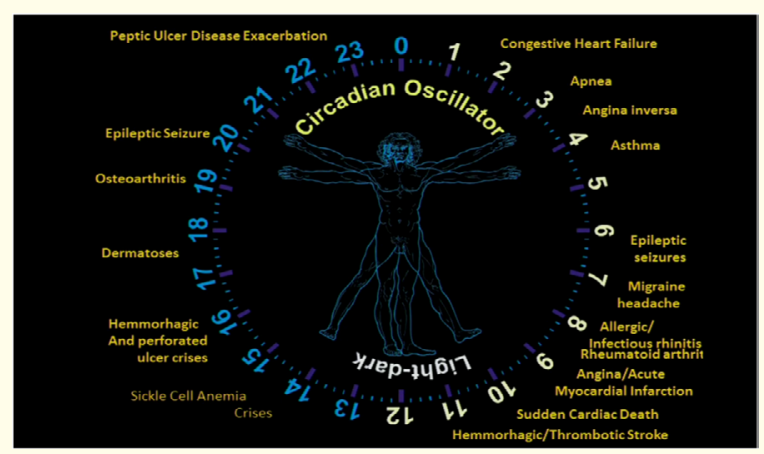

Figure 13

\section{Resetting our biological clock with light}

So that the circadian pacemaker remains synchronized with the changing daylight during the different seasons, it is continuously readjusted in the corresponding direction, so it can advance or delay its phase.

Although light is the most potent stimulus to adjust the circadian system, other factors can also do it, such as activity schedules, sleep, eating, and some drugs as melatonin.

Each person has a different circadian rhythm, and we can find cases that last between 23.5 and $24.6 \mathrm{~h}$, being the average of $24.2 \mathrm{~h}$.

In addition, our biological clock can be reset only by managing the intensity and color of the light in a single day between 22 and 27h. In this way, we can solve problems such as Jetlag or night work shifts. As we can see, it is easier to extend our biological day than to shorten it.

The mismatch of our biological clock concerning the dominant light/dark cycles in our activity can produce severe health problems, wellbeing, alertness, efficacy, and safety at work. 
To readjust our circadian system, we can work with the following characteristics of light: Moment of exposure, intensity, duration, pattern, the color of the light (wavelength), and recent exposure history.

Next, we will analyze each of these characteristics separately, although it is the set of all of them that can lead us to achieve the strongest adjustments.

Moment of exposure is one of the most critical factors for the readjustment of the circadian phase is the time when exposed to light is performed. Under normal conditions, when the exposure to light is done in the afternoon/evening, resulting in a phase and sleep delay. If the exhibition is held in the early morning or early morning, the phase is advanced. At noon, exposure to light is ineffective for phase changes. By correctly choosing the time of day for exposure to light, we can influence to solve sleep problems.

There is a non-linear relationship between light and its intensity to readjust the phase of our circadian system. If we expose ourselves inside a building to relatively low levels of light of, for example, 100lux for 6.5 hours at night, we are stimulated 50\% more compared to being exposed to 10lux during the same time.

The duration and pattern of exposure to light also have a nonlinear influence. Short and time-distributed exposures affect us comparatively more than long continuous periods. The results obtained were compared to a continuous $6.5 \mathrm{~h}$ light exposure at night, with a discontinuous exposure so that every 60 minutes it was exposed only for 15 minutes at the same level of light in both cases. The result is that in the first one, the lag was 3 hours, and in the second 2.5 hours. In other words, the latter case obtained an $80 \%$ response with respect to the first using only $23 \%$ of the time.

If we compare the effectiveness of the monochromatic wavelength of $480 \mathrm{~nm}$ with that of $550 \mathrm{~nm}$, the first is twice as effective as the second to cause a phase change. We need precision to measure the circadian effect.

Historical exposure, the effects of light stimuli in the circadian system in addition to being affected by intensity, it is also due to variations in said intensity over time. The circadian photo-reception system shows adaptation to previous light exposure to determine the effect of the current exposure. If we have received little radiation in the last hours, any amount of radiation will have a significant impact. If we have received a lot of light radiation during the last hours, the effect of the additional radiation that we are receiving at this moment diminishes very significantly.

For more details at this point consult the note [32].
Lux and Lumen are not suitable for measuring the non-visual effects of light

Lux and Lumen are metrics designed for vision, being inadequate to estimate the non-visual effects of light.

We need additional metrics to quantify the radiation perceived by the eye and to estimate the non-visual effects of light.

Scientists and standardization authorities are working to define the new metrics in which the important thing is the amount of light entering the eye and its spectral distribution (the colors of light) with peak sensitivity in blue. The light intensity measurements will not be performed on the work plane as is the case to estimate the visual effects but will be performed perpendicular to the eye.

We will continue to use the Lux to estimate the visual effects. To determine the non-visual impact, we will use the Melanopic Equivalent Daylight Illuminance (MEDI) metric.

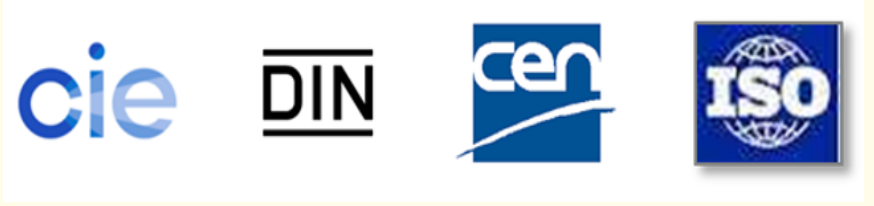

Figure 14

We have evolved under the dark light cycle produced by the rotation of the earth. Light is the main stimulus our body needs to activate and synchronize with the 24 -hour cycle. In the table below we can see the sensitivity of our circadian system to the "mel" light and our "V" vision system for the illuminant D65. Since daylight is different depending on the time, the season of the year and the geographic location of the observer, they have established the illuminant D65 as the reference light.

There is not such a D65 light source. It is only a mathematical function that reproduces the spectral characteristics of outdoors daylight in Brussels at noon in June. It includes both direct and diffused sunlight in a clear sky. It has a correlative color temperature of approximately 6,500 K. The standard D65 illuminant should be used for comparison in all colorimetric calculations that require daylight representation.

Scientists have been able to measure that to activate and synchronize our biological clock with the natural light/dark cycle we have to receive the equivalent of 240 MEDI of the standard D65 light source preferably in the morning.

As the transmission of light to our retina is reduced by the darkening and color change of the lens with age. 


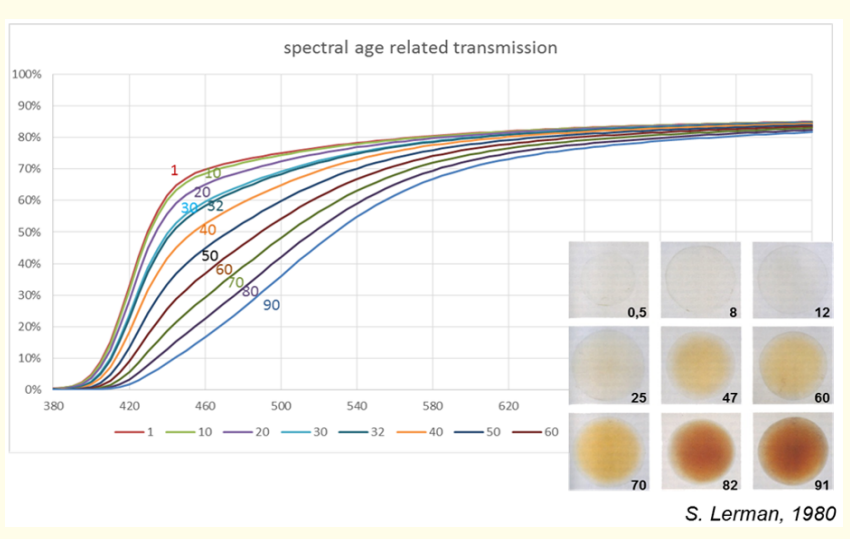

Figure 15

We have to increase the amount of MEDI that the elderly receives to have the same circadian impact as a 32-year-old person who is the reference.

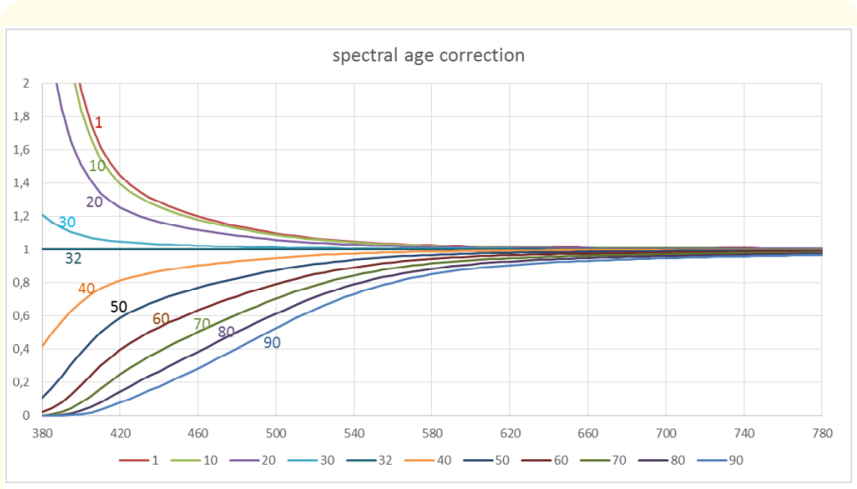

Figure 16

A 32-year-old person will need 240 MEDI to activate, and a 75-year-old person will need 519 MEDI to have the same impact.

In figure 17, we can see an equivalence table between the Lux and melanopic Lux of different light sources.

\begin{tabular}{|c|c|c|c|}
\hline Illuminant & $\begin{array}{c}\text { Photopic } \\
\text { illuminance } \\
\qquad E_{V}\end{array}$ & $\begin{array}{c}\text { Melanopic } \\
\text { daylightequivalent } \\
\text { illuminance } \\
E_{\text {melDbs }}\end{array}$ & $\begin{array}{l}\text { Conversion factor } \\
m_{\text {vmelDos }}=E_{\text {melDos }} / E_{V}\end{array}$ \\
\hline Std. illuminant $A(C C T=2856 \mathrm{~K})$ & $1000 \mathrm{~lx}$ & $496 \mathrm{~lx}$ & 0,496 \\
\hline Fluorescent type CIE F10 (FL, CCT $=5000 \mathrm{~K})$ & $1000 \mathrm{Ix}$ & $683 \mathrm{Ix}$ & 0,683 \\
\hline Fluorescent type CIE F12 (FL, CCT $=3000 \mathrm{~K})$ & $1000 \mathrm{Ix}$ & $404 \mathrm{Ix}$ & 0,404 \\
\hline Std. illuminant D65 (daylight CCT=6500 K) & $1000 \mathrm{Ix}$ & $1000 \mathrm{Ix}$ & 1,000 \\
\hline illuminant $P$ (candle light) & $1000 \mathrm{Ix}$ & $267 \mathrm{Ix}$ & 0,267 \\
\hline LED, white (CCT $=3035 \mathrm{~K})$ & $1000 \mathrm{~lx}$ & $428 \mathrm{Ix}$ & 0,428 \\
\hline LED, white (CCT $=5400 \mathrm{~K}$ ) & $1000 \mathrm{~lx}$ & $787 \mathrm{Ix}$ & 0,787 \\
\hline LED, white (CCT $=6535 \mathrm{~K})$ & $1000 \mathrm{~lx}$ & $8001 x$ & 0,800 \\
\hline High CCT Fluorescent (CCT= $8000 \mathrm{~K})$ & $1000 \mathrm{~lx}$ & $957 \mathrm{Ix}$ & 0,957 \\
\hline
\end{tabular}

Figure 17
If the lighting we receive comes from a 3000k fluorescent light source, we would need $1000 / 0.404=2475$ lux. Or what is the same, to get the equivalent of 240 MEDI of the D65 source we would need 595lux of a fluorescent light source of $3000 \mathrm{k}$.

The human immune response is circadian

- In the absence of light and especially circadian blue light (around 480nm), Th1 cells produce immune activity through $\mathrm{T}$ cells and $\mathrm{N}$ antitumor cells.

- With the presence of circadian light during the day, Th2 cells produce humoral immune response through B cells reacting against pathogens.

When we reduce to 4 hours our sleep, we cut up to $70 \%$ the production of $\mathrm{N}$ killer cells. When our circadian system is in sync, the risk of cancer and infectious diseases decreases.

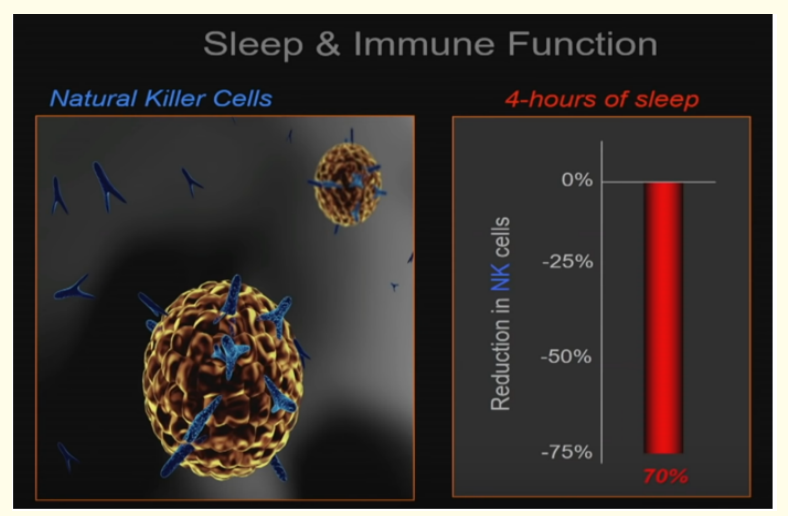

Figure 18

When our circadian system is NOT in sync, the risk of disease is increased, we have sleep problems. We can have migraines and visual problems, and we increase appetite, we have more confusion with less coordination, discomfort, and sadness increases.

Electric light was applied to allow people to live and work in environments with poor natural lighting, in short, to extend the time of human activity and to improve living conditions.

In some cases, we are neglecting these concepts for the sake of cost reduction. We do not put in the fundamental balance aspects such as health, comfort, well-being, or the effectiveness of people.

Buying a cheap and inadequate luminaire is a decision that is made in a minute, but its effects are permanent to the users for many years.

One hundred thirty-six years ago, the invention of electric light meant a revolution. We replaced the chandeliers and candles that 
polluted a lot with a more efficient electric lamp, much safer, ecological, and economical in their total cost. Today, we are witnessing a new revolution based on new scientific knowledge about how our body works and the application of new lighting technologies working in unison.

In 1929 the French architect Le Corbusier said 'the history of architecture has been characterized by an endless struggle for light... in other words, the struggle for windows. ' Most architects think that daylight is very important for people, and consequently, few architectural components have more influence on their designs. However, so far, it has not been able to take full advantage of this design effort.

Today we already have solutions to more effectively illuminate the interiors of buildings both from the roof and from the windows.

Most of us prefer to be near the windows whenever we have thermal and visual comfort.

\section{Nighttime shift work [33]: a problem to be solved}

During working hours at night, we have low light levels with low blue content. Our brain interprets that is time to sleep. As we go home, we have bright natural light in the street, and our brain interprets is time to wake up. Sleep will be shorter and increasing our sleep debt making it harder to adapt to the night shift work.

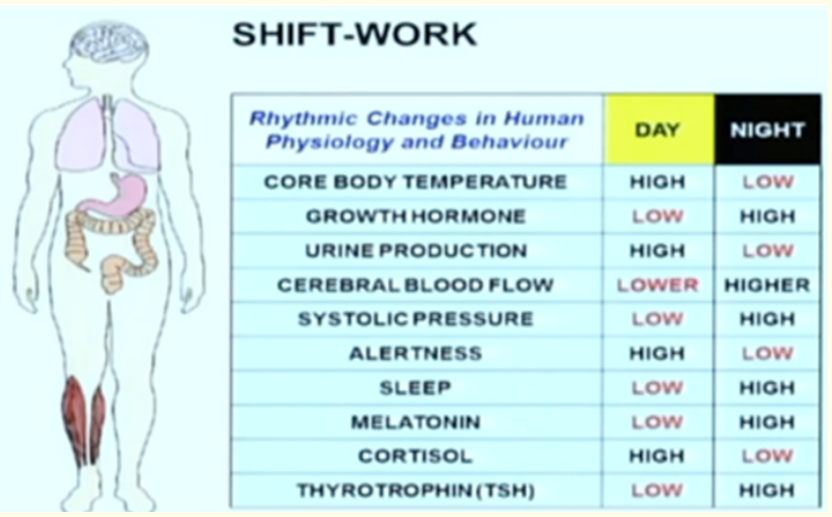

Figure 19

Sedation by substances is not the same as natural sleep. Sedatives will affect creativity and memory.

\section{Lighting in health care buildings}

In the design of healthcare facilities, medical professionals, architects, and engineers are increasingly realizing the importance of creating a healthy environment that addresses the patient and staff biological needs. This more holistic approach is driven by the recognition that a patient's perception of the physical environment in a hospital can affect his or her sense of wellbeing and, potentially, health.

The hospital sector requires special consideration as inhabitants are either sick, chronically ill, or disabled in basic daily activities. They may be with or without visual problems, frailty, movement disorders, sleep disturbances or memory and cognitive problems. Light can play a very prominent role in improving the quality of life for these individuals. Encouraging more use of outdoor spaces has a significant impact if used in association with suitable lighting indoors. The eye's health (lens transmission, pupil size, and reflex) can be measured and assessed. Accurate information is essential for vision (glare, acuity) and non-image forming function (sleepwake timing) and must be taken into consideration for lighting solutions.

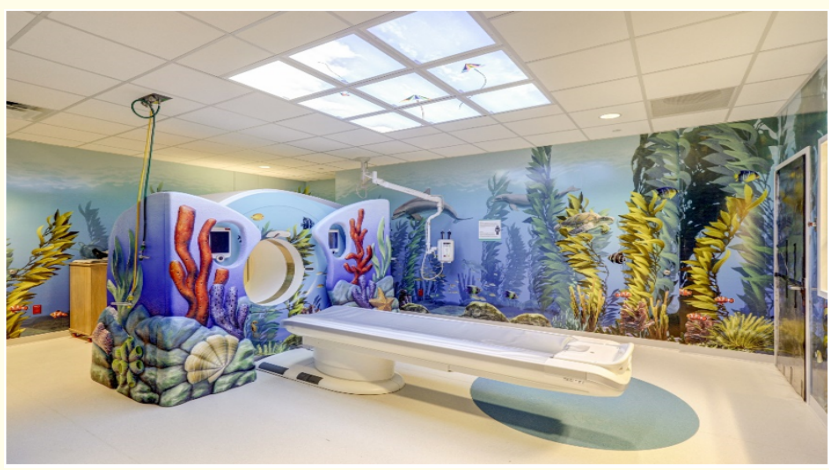

Figure 20

The temporal 24-hour light-dark cycle becomes very important for the repair and regeneration of cells because cell cycles are dependent on strong signals from the body clock, whose activity is influenced by light quality, intensity, and timing. Therefore, personalized dynamic light settings for each room in hospitals should be propagated. For example, dynamic light can include a dawn simulation, a spectrum of light that fluctuates with warmer color temperature over the course of the day, and an absence of cool color temperature in the evening. Furthermore, in a hospital, if possible, a patient should be allowed to adjust light levels.

Patients should be able to control the light settings in the room. This will support the healing process and enhance the quality of life for them.

The design of lighting systems in nursing homes are made to support visual acuity for staff and to minimize hazards such as staircases. However, to obtain proper visual sharpness and better contrast, people of older age require heightened light levels due to an age-related reduction in visual capability. 
Also, more light during the day will result in better sleep during the night for the inhabitants. The nursing home environments are often purpose-made for hygiene, cleanliness, and safety and ignore that light sources produce substantial glare. That inappropriate light at night disrupts not only sleep but also the timing of the body clock, with negative consequences for cognition, emotions, and wellbeing. Properties of current lighting systems are inflexible and not designed to take non-image forming effects of light into account for patients or older people's wellbeing in hospitals or nursing homes.

In addition to meeting the age-related demands with increased illumination, aspects of light's non-image forming effects on mood, cognition, alertness, sleep, and internal rhythms, can and should be implemented in future designs of healthcare buildings. In particular, those of daylight exposure and dynamic lighting systems need to be considered. Ideally, building and indoor designers need to consider the direction of natural light, glass structures, and proportions of window surfaces to allow maximizing natural light exposure. Specialized lighting solutions for individual rooms with different purposes (recovery or emergency rooms in a hospital, bedrooms or day-activity rooms in nursing homes), should be programmed to be remotely user-tunable by staff, patient, resident or caregiver. Being in control of the environment increases independence, confidence, and a positive attitude in individuals that are beneficial for the mood and any healing process.

Peer-reviewed $[34,35]$ researches in healthcare buildings have demonstrated that access to daylight provides;

- Reduction in the average length of hospital stay (Shorter $14-41 \%$ in SE rooms compare to NW).

- Quicker post-operation recovery and reducing ICU delirium.

- $\quad$ Reduced requirements for pain relief (The patients are receiving $46 \%$ more natural sunlight, required $22 \%$ fewer analgesic medications during hospitalization).

- Quicker recovery from depressive illness (morning light is twice as effective as evening light in treating SAD seasonal affective disorder).

- There is also a growing body of evidence that daylight plays a critically important role in the prevention and treatment of obesity, heart disease, and other illnesses exacerbated by stress.

The most successful hospitals are those that focus on the health and well-being of patients, health professionals, and the families visiting the patients.

Much of this can be achieved by adapting the indoor environment (light, sound, welcoming views) to match the circadian response and to reduce stress in all three participants. Proper light-

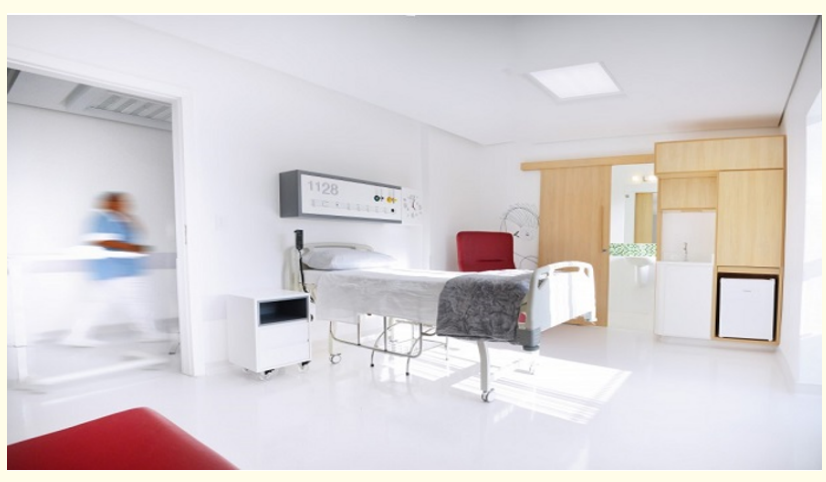

Figure 21

ing that incorporates visual and circadian stimuli, noise reduction strategies, stress-reducing views (windows overlooking gardens, or pictures of gardens) have been shown to improve patient recovery, productivity in hospital personnel and stress reduction in patient and visitors.

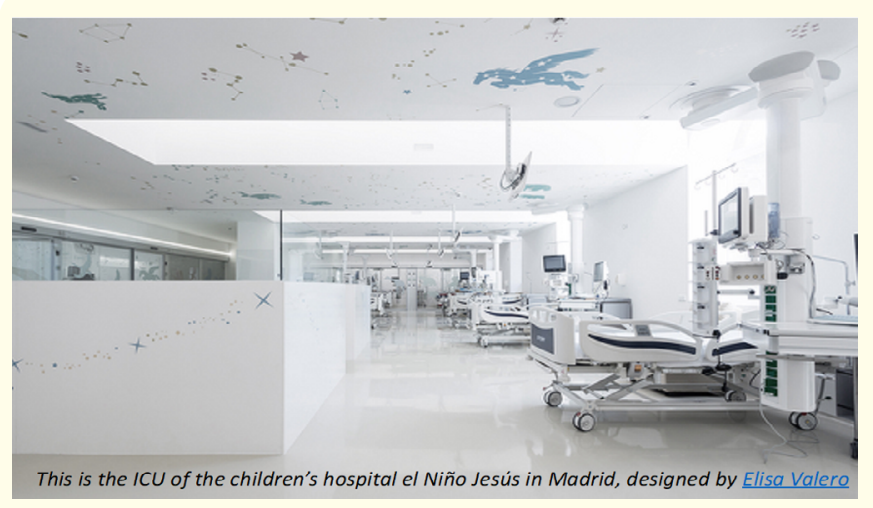

Figure 22

In neonatal NICU [36], the lights are usually lit 24 hours a day, and this deteriorates the unmatured functioning of the circadian system of the newborn. By covering the children with a cloth for 12 hours a day, they get a $50-60 \%$ increase in oxygen saturation in the blood. The weight of the babies increases dramatically, and they leave the NICU 5 weeks earlier compared to the control group.

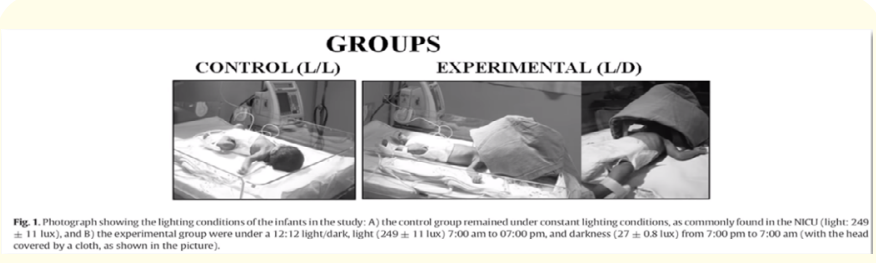

Figure 23

Human Centric Lighting is already making its way either through newly built buildings or by means of renovation. Such installations can be introduced in a variety of application fields in care buildings, 


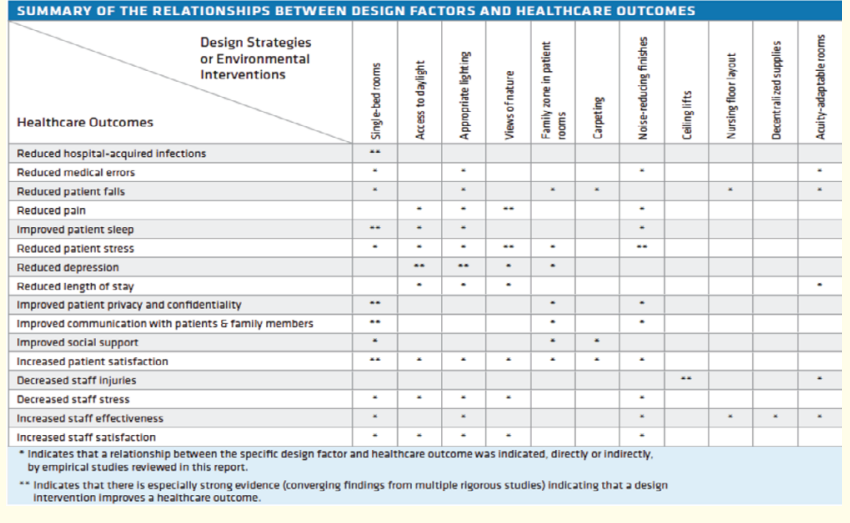

According to IES RP-29-26-revised

Figure 24

such as offices areas, UCI, operating rooms, rooms, waiting rooms, corridors, so in general in hospitals, and nursing homes. Depending on the activity to be performed, there are specific types of lighting that need to be applied.

In the document of the Eindhoven University of Technology "Measurements of ICU Environment" we can read; Pharmacological assistance alone cannot achieve the desired quantity and quality of sleep in ICUs, not to mention its detrimental side effects. ICU environments can enhance the healing process if relatively simple stress-reducing sleep improving elements are incorporated into their physical design. Interventions have been developed to reduce noise and disruptive staff-patient interactions at night. Also, to improve lighting and offer privacy, to provide positive distractions such as contact with nature, or to maintain the daily lightdark cycle. They've shown favorable results. Focus, however, has been on the acoustic environment, ignoring the rest of the physical environment.

ICU patients must have delta (deep) sleep. Sounds and inappropriate light will prevent delta sleep. Delta sleep is "healing sleep" with an increase in neurotransmitters for wound healing.

\section{Seeing is believing}

We suggest to make a "mock-up " in a room or an area of a hospital with the proper lighting including circadian stimulation during the day, no direct lighting above the patients eye when laying down; blocking or lowering of beeping sounds of instruments, and a patient view out of a window or a soothing landscape (ocean waves etc.). No light at night or only red light for guidance to the bathroom and back to bed. Reduce as much as possible visits from the care team using the same red light if possible and if required, only no glaring and focused worm light. All this has to be done together and in agreement with the medical team if not, the project will fail.
Allow your visitors to "experience" the physiological difference when the environment of the room is modified. And most importantly, measure the outcomes of the new installation.

A very interesting exercise would be to check the length of stay of your patients in the hospital comparing those staying in southfacing rooms with those facing north-facing rooms ii You will be surprised with your findings!! Our ipRGC sensors do not differentiate if the received light comes from a natural source or from an electric one. If you want to have the same results in both room types, you just need to compensate the lack of natural light in north face rooms with a full spectrum electric light. The amount of light with a full spectrum happen to make the differences.

The art of good lighting design can be used to create a welcoming, emotionally supportive environment that lower anxiety. Patients who are able to reduce psychological stress levels and who receive strong emotional support from visitors and caregivers have been shown to exhibit quicker wound recovery according to Gouin JP, and Glaser JK. The impact of phycological stress on wound healing.

Daylight deprivation in buildings has been shown to have hugely damaging consequences. Without access to daylight, human bodyclock becomes disrupted. It needs recalibration daily, and unless we receive adequate daylight, overwhelming medical evidence suggests that humans become stressful and agitated. Any disruption to our circadian rhythm has highly negative consequences in terms of our health, happiness, and wellbeing.

As daylight is not available when required, we have to mimic its characteristics with electric lighting in amount, timing, direction, and spectrum quality.

\section{Conclusion}

It is relevant to note that people's centricity is a key strategy for improving their wellbeing and productivity in indoor environments. Nevertheless, this is only achievable if smart spaces are developed and contribute to physical health and comfort of users. Human-Centric Lighting is one of the key issues that have an essential contribution to the wellbeing of healthcare workers and patients, supporting patient health wellbeing and safety.

The immune system is a sleep instigator, as a result of being infected, our body is sleepier to promote healing, but people sleep less in hospitals. The organization of the hospitals [37] makes a deep and restful sleep difficult.

The new scientific knowledge has the potential to change our lives for the better iiilet's make it happen in hospital environments!!! 


\section{Sleep is NOT an option}

The Synergy of Light and Life.

More reading about circadian and health

1. Intrinsically photosensitive retinal ganglion cells: many subtypes, diverse functions. Tiffany M. Schmidt1

2. DIN SPEC 67600-Biologically effective illumination-Design guidelines.

3. The Genetic Basis of Sleep and Sleep Disorders by Paul Shaw, Mehdi Tafti and Muchael Thorpy, published by Cambridge Medicine

4. Why We Sleep by Matthew Walker, published by Penguin

5. Breast cancer and circadian disruption from electric lighting in the modern world Richard G. Stevens PhD, George C. Brainard PhD, David E. Blask PhD, MD Steven W. Lockley PhD Mario E. Motta MD

6. Circadian regulation of molecular, dietary, and metabolic signaling mechanisms of human breast cancer growth by the nocturnal melatonin signal and the consequences of its disruption by light at night David E. Blask, Steven M. Hill, Robert T. Dauchy, Shulin Xiang, Lin Yuan, Tamika Duplessis, Lulu Mao, Erin Dauchy, Leonard A. Sauer

7. Disrupting the circadian clock: Gene-specific effects on aging, cancer, and other phenotypes Elizabeth A. Yu, David R. Weaver

8. Melatonin: clinical relevance Russel J Reiter PhD

9. Circadian clock control of the cellular response to DNA damage Aziz Sancar, Laura A. Lindsey-Boltz, Tae-Hong Kang, Joyce T. Reardon, Jin Hyup Lee, Nuri Ozturk

10. How our body clocks can make or break our health Maria Cohut, Medical News Today

11. Could our body clock help to prevent cancer? Medical News Today

12. How night shifts can increase cancer risk Honor Whiteman, Medical News Today

13. Using the body clock to make drugs more effective Ana Sandoiu, Medical News Today

14. Could targeting the body clock stall brain cancer? Maria Cohut, Medical News Today

15. Lighting Europe. http://www.lightingeurope.com

16. licht. wissen 21 Guide to Human Centric Lighting (HCL).

\section{Bibliography}

1. Russell G Foster and Leon Kreitzman. "The rhythms of life: what your body clock means to you!". Experimental Physiology 99.4 (2014): 599-606.
2. Robert Lucas. "Re-imagining vision following the discovery of new photoreceptors".

3. S W Lockley. "Circadian Rhythms: Influence of Light in Humans Brigham and Women's Hospital and Harvard Medical School". Boston, MA, USA.

4. http://www.bio.brandeis.edu/faculty/hall.html

5. http://www.bio.brandeis.edu/rosbash-lab/index.php/publications/

6. B Philip., et al. "Temperature compensation and temperature sensation in the circadian clock". Proceedings of the National Academy of Sciences of the United States of America 112.46 (2015): 6284-6292.

7. T Anand., et al "Flavin reduction activates Drosophila cryptochrome". Proceedings of the National Academy of Sciences of the United States of America 110.51 (2013): 20455-20460.

8. Helena J Bailes and Robert J Lucas. "Human melanopsin forms a pigment maximally sensitive to blue light (lmax $479 \mathrm{~nm}$ ) supporting activation of Gq/11 and Gi/o signalling cascades". Proceedings Biological Sciences 280.1759 (2013): 20122897.

9. Robert J Lucas., et al. "Measuring and using light in the melanopsin age”. Trends in Neurosciences 37.1 (2014): 1-9.

10. Daylight in buildings ILNAS-EN17037 (2018).

11. Deroisy B and Deneyer A. "A new standard for daylight: Towards a daylight revolution?" Department Acoustics, Energy and Climate Belgian Building Research Institute, BBRI Limelette, Belgium (2017).

12. Lighting Europe and IALD define HCL.

13. CIE.

14. Roberts JE. "Ocular Phototoxicity". Journal of Photochemistry and Photobiology B 64.2-3 (2001): 136-143.

15. Shang-Ru Tsai and Michael R Hamblin. "Biological effects and medical applications of infrared radiation". Journal of Photochemistry and Photobiology 170 (2017): 197-207.

16. Maria Cohut. "How our body clocks can make or break our health". Fact checked by Paula Field (2018).

17. Roberts JE. "Light and Immunomodulation". New York Academy of Sciences 917 (2000): 435-445.

18. https://iimel.es/que-es-la-melatonina

19. Gustavo Tamosiunas and Mauricio Toledo. "Chronopharmacology: a new variable that could account for the variability of the therapeutic response". 
20. Azure D Grant and Gary I Wolf. "Free-Living Humans Cross Cardiovascular Disease Risk Categories Due to Daily Rhythms in Cholesterol and Triglycerides" (2019).

21. Chrono Pathology

22. Reza Kazemi., et al. "Effects of Shift Work on Cognitive Performance, Sleep Quality, and Sleepiness among Petrochemical Control Room Operators". Journal of Circadian Rhythms 14 (2016): 1 .

23. with the lack of sleep

24. Steven W Lockley., et al. "Effect of Reducing Interns' Weekly Work Hours on Sleep and Attentional Failures". The New England Journal of Medicine 351.18 (2004): 1829-1837.

25. Micheline Maire., et al. "Fighting Sleep at Night: Brain Correlates and Vulnerability to Sleep Loss". Annals of Neurology 78.2 (2015): 235-247.

26. Bernsmeier., et al. "Sleep Disruption and Daytime Sleepiness Correlating with Disease Severity and Insulin Resistance in Non-Alcoholic Fatty Liver Disease: A Comparison with Healthy Controls". Plos One 10.11 (2015): e143293.

27. Steven W Lockley., et al. "Effect of Reducing Interns' Weekly Work Hours on Sleep and Attentional Failures". The New England Journal of Medicine 351.18 (2004): 1829-1837.

28. Julia ML Menon., et al. "Brain Microdialysate Monoamines in Relation to Circadian Rhythms, Sleep, and Sleep Deprivation - a Systematic Review, Network Meta-analysis, and New Primary Data" Journal of Circadian Rhythms 14 (2019): 17.

29. Bryony Sheaves and Colin Espie. "Having trouble with your sleep? Try following these 10 top tips for better sleep". University of Oxford.

30. Brigham and women's hospital and Harvard medical school

31. Joan E Roberts. "Circadian Rhythm and Human Health. Department of Natural Sciences".

32. https://blog.lsgc.com/wp-content/uploads/2016/04/14.2009-Lockley-Encyclopedia-Neurosci-Book-Chapter.pdf

33. Clare Anderson., et al. "Deterioration of Neurobehavioral Performance in Resident Physicians During Repeated Exposure to Extended Duration Work Shifts". Sleep 35.8 (2012): 11371146.

34. DTG Strong. "Daylight benefits in healthcare buildings".

35. Kathleen M Beauchemin and Peter Hays. "Dying in the dark: sunshine, gender and outcomes in myocardial Infarction". Journal of the Royal Society of Medicine 91.7 (1998): 352-354.
36. Mirmiran M and Ariagno RL. "Influence of light in the NICU on the development of circadian rhythms in preterm infants". Seminars in Perinatology 24.4 (2000): 247-257.

37. Matthew. "Walker on Sleep for Enhancing Learning, Creativity, Immunity, and Glymphatic System".

Volume 3 Issue 11 November 2019

(C) All rights are reserved by Rafael Lledó., et al. 